\title{
Comparing Pointing Techniques for Grasping Hands on Tablets
}

\author{
Katrin Wolf \\ University of Stuttgart \\ katrin.wolf@vis.uni-stuttgart.de
}

\author{
Niels Henze \\ University of Stuttgart \\ niels.henze@vis.uni-stuttgart.de
}

\begin{abstract}
With the recent success of tablet devices a new device type became available for mobile interaction. Just as for mobile phones, touch is the dominant way people interact with tablets. In contrast to the much smaller phones a firm grip with both hands is needed to securely hold tablet devices. While a large body of work has investigated touch interaction on smaller devices, is little empirical research has been carried out on touch-based pointing while holding the device with both hands. To understand touch-based interactions using tablet devices, we conducted an experiment to compare four pointing techniques on both the front and back of the devices while it was held in landscape format. We compare direct touch with the following alternatives for selecting targets, indirect pointing on a virtual touchpad, an inverse cursor, and a miniature interaction area. While direct touch is $35 \%$ faster than the fastest alternative, only $74 \%$ of the touchscreen and $64 \%$ of a back-of-device can be reached by each hand. We show that among the indirect pointing techniques, the miniaturized interaction area is significantly faster and received the best subjective ratings. We conclude that a miniaturized interaction area is a viable alternative to direct touch especially on the backside of tablet devices.
\end{abstract}

\section{Author Keywords}

Tablet; pointing; touch; direct; indirect; grasp.

\section{ACM Classification Keywords}

H.5.2. User Interfaces: Ergonomics.

\section{INTRODUCTION}

Over the last decade, touchscreens have arguably become the most common way to interact with computing devices. A large body of work has investigated how mobile devices with touchscreens are used in daily life and, in particular, developed novel interaction techniques for them. As direct manipulation is the dominant interaction paradigm for touch devices, investigating pointing techniques received major attention. Previous work has focused either on the interaction with mobile phones that can easily be held in

Permission to make digital or hard copies of all or part of this work for personal or classroom use is granted without fee provided that copies are not made or distributed for profit or commercial advantage and that copies bear this notice and the full citation on the first page. Copyrights for components of this work owned by others than ACM must be honored. Abstracting with credit is permitted. To copy otherwise, or republish, to post on servers or to redistribute to lists, requires prior specific permission and/or a fee. Request permissions from permissions@acm.org.

MobileHCI 2014, September 23-26, 2014, Toronto, ON, Canada.

Copyright (C) 2014 ACM 978-1-4503-3004-6/14/09..\$15.00. one hand (leaving the other hand free to interact) or on interaction with a much larger stationary touch display.

With the commercial success of tablet computers a third category of touch screen devices has become widely used. While tablets are small enough to be used "on the go" they are often too large to be held comfortably with one hand.

Indeed one of the most common ways to hold the device is using a two-handed grip (particularly if used in landscape format). Grasp-based pointing on tablets can cause ergonomic problems. Due to the large size of tablets, the thumbs and fingers are sometimes unable to reach the center of the display, while the device is being held [12, 21]. Therefore, direct touch, which is the most common pointing technique for touchscreens, may be not the most appropriated one for tablet devices.

The technology company Apple filed a patent on back-ofdevice interaction in 2006 [9]; and Wigdor et al. [20] demonstrated in 2007 how back-of-device interaction when using a tablet-sized device solves the fat-finger problem [16]. Since the release of the Motorola CHARM in 2010 and the Sony Playstation Vita in 2012, mobile devices with a touch-sensitive back are commercially available. It can be expected that touchpads will soon be embedded in the back of many devices. Users' performance when pointing through back-of-device interaction on tablet has not been study in-depth, and consequently, corresponding design guidelines are not available. Thus, we consider both, pointing on touchscreens as well as on touchpads built in the back of the device.

Pointing performance with grasping hands is constrained by parameters, such as reachability and joint flexion of the pointing digit. To understand touch-based interaction using tablet devices, we conduct an experiment that compares four pointing techniques on the front and on the backside of tablet devices while the device is being held in landscape format. We compare direct touch with selecting targets using a touchpad, an inverse cursor, and a miniature interaction area.

The contribution of this paper is threefold: (1) Describing the area that is easily accessible using direct touch while the tablet is held in landscape format based on empirical data. (2) Comparing four pointing techniques on both sides of tablet devices to show which technique is the best alternative to direct touch. (3) Proposing a combination of direct touch on the front and a miniature representation of 
the tablet's interaction area on the back that makes the whole screen accessible.

In the following sections we first provide an overview about previous work on pointing techniques. Afterwards, a description of the conducted study and its results is given. Finally, we discuss our findings and conclude the paper with a summary and an outlook on future work.

\section{RELATED WORK}

This paper aims to increase our understanding of pointing on tablets. The aim is to overcome the current limitations caused by occlusion and limited target accessibility in the center of the device. Research with respect to pointing on tablet devices is rare. Thus we present here works on pointing with hand-held devices in general, which includes mobile phones. We identified four categories of pointing techniques: direct and relative pointing, remote direct pointing, and inverse direct pointing, which are presented in the following sections.

\section{Direct pointing}

Direct pointing is known to be very immediate and intuitive as touching the desired target corresponds directly to the way people interact in the physical world. In contrast to the physical world however, virtual targets are often very small. This can result in a phenomenon known as the fat-fingerproblem [16], which means that the finger that touches the target is occluding it, thereby decreasing precision. LucidTouch [20] is a tablet-sized device that enables backof-device interaction; and thus the fingers can select targets from the back side without occluding the content that is displayed on the front. LucidTouch uses a camera mounted at the rear of the device, which results in a rather bulky prototype. The concept of back-of-device interaction has been improved by Baudisch and Cheng: NanoTouch [1] uses a back-mounted touchpad instead of a camera and thus, enables back-of-device interactions even with very small devices.

If a user holds the device while pointing, the hand has to solve multiple tasks, meaning that direct pointing becomes more challenging due to the hands bio-mechanics. Thus, in addition to occlusion, a second problem of direct touch is the accessibility of targets that are further away or very close. The center of the tablet is hard to reach if the device is held in landscape format with both hands [12, 21]. For one-handed pointing on mobile phones it was found that the thumb performance varies with its posture. Poorest pointing performances results from excessive thumb flexion. When tapping on targets closest to the base of the thumb in the bottom right corner of the screen the performance is low. The highest performance is achieved when the thumb is in a rested posture, neither significantly flexed nor fully extended [17]. De Luca et al. [5] showed that back-ofdevice pointing without visual feedback has a too low accuracy to be considered a valuable input method for authentication. Finally, Buschek et al. developed a model to improve text entry on the back of the device but did not compare direct touch with other pointing techniques [2].

\section{Inverse direct pointing}

Roudeau et al. [14] introduced MagStick, which is a thumb interaction technique for target acquisition on mobile devices with small touch-screens. The technique addresses screen accessibility as well as target selection accuracy and occlusion. The user controls a cursor through an inverse drag motion and thus can select a target without occluding it with the thumb. While MagStick enables to access a larger area than the thumb can reach via direct touch, Roudeau et al. found that it is slower.

Kim et al. [10] proposed an expandable cursor called Large Touch that also moves inversely to the user's finger. In contrast to MagStick, the cursor moves a larger distance than the thumb that slides across the touchscreen. Thereby, Large Touch enables to reach locations that are further away from the thumb. Also in contrast to Roudaut et al. [14], Kim et al. found no difference in target selection time between the inverse cursor technique (Large Touch) and the common direct touch technique in a conducted study [10].

\section{Miniature interaction area}

ThumbSpace [8] introduced one-handed thumb interaction for small targets that are spread out wide on mobile phones' screens. The problem addressed is similar to the problem of pointing on targets that are hard to reach due to the size of a tablet. The approach taken is to shrink the screen into a small screen that is defined by drawing it with the thumb. This ensures that the thumb can reach all targets. Thus, ThumbSpace improves accuracy for selecting targets that are out of thumb reach, but it is slower than target selections with direct touch. A similar concept was proposed by Kim et al. [10] who presented Sliding-screen to address the limited target accessibility on phone touchscreens. A drag from the edge of the screen towards its center dynamically shrinks the interaction area, meaning that a tap can easily reach targets on the smaller display that may have been too far away on the interaction area before it was shrunk. Like ThumbSpace, this technique was found to be slower than direct touch for one-handed target selections with mobile phones.

The ARC-Pad [11] links the touchscreen of a phone to a large display in a one-to-one mapping. It enables to use the phone's touchscreen to be used as both an absolute and a relative touchpad for large displays. ARC-Pad combined absolute and relative cursor positioning. Tapping on the $A R C$-Pad causes the cursor to jump to the corresponding location on the screen, providing rapid movement across large distances. For fine position control, users can use a relative cursor control technique.

\section{Relative pointing}

Relative pointing is often used for remote-selections, such as mouse and touchpads that are built in laptops. Forlines et 
al. [6] compared direct touch versus mouse input for unimanual and bimanual tasks on tabletop displays. Analyses of quantitative performance and subjective preference indicate that users may be better off using a mouse for unimanual input and their fingers for bimanual input when working on a large, horizontal display. Cockburn et al. [4] compared performance in touch selections on a touchscreen that was horizontally placed on a table in front of the user. They found that direct touch is faster than relative pointing (tap is faster than drag) using the finger. The error rate is high for small targets and further increases using direct pointing methods for target acquisitions over longer distances.

Hasan et al. compared relative pointing with direct touch for back-of-device interaction [7]. They found that relative pointing is faster and more accurate on the back of the device.

A comparison of ARC-Pad [11], which was described above, with relative pointing showed that ARC-Pad is faster than relative pointing. Moreover, relative pointing was more accurate. Thus, the ARC-Pad was, just like direct touch usually is, namely faster but less accurate than relative pointing.

\section{Summary}

Previous work developed and compared pointing techniques for tabletop displays $[4,6]$, for mobile phones' touchscreens $[8,10,11,14]$, and for the backside of phones [7]. Tablets are, however, a third device type that has almost been entirely neglected. Their size/weight requires a certain grip that not only affects pointing performance but also makes parts of the screen inaccessible using direct touch. In the following sections we reduce this research gap through a controlled experiment. Four target pointing techniques, which represent the four categories discussed above, are compared. We consider front- as well as backof-tablet interaction using both the dominant and the nondominant hand. The aim is to provide a fundamental understanding of pointing on tablets with grasping hands.

\section{POINTING TECHNIQUES}

Previous work proposed the following four categories of touch-based pointing techniques: direct pointing, inverse direct pointing, relative pointing, and remote direct pointing. In our experiment, we evaluate four pointing techniques (see Figure 1); and each of them represents one of the categories. Direct pointing is represented through direct touch (1). The inverse cursor (2) stands for the direct inverse pointing techniques. The touchPad (3) allows relative pointing; and the miniature area (4) enables direct remote pointing using a miniaturized display that represented the entire interaction area of the tablet.

\section{(1) Direct touch}

Direct touch is the common pointing technique for touchscreen interactions. The digit is directly touching the

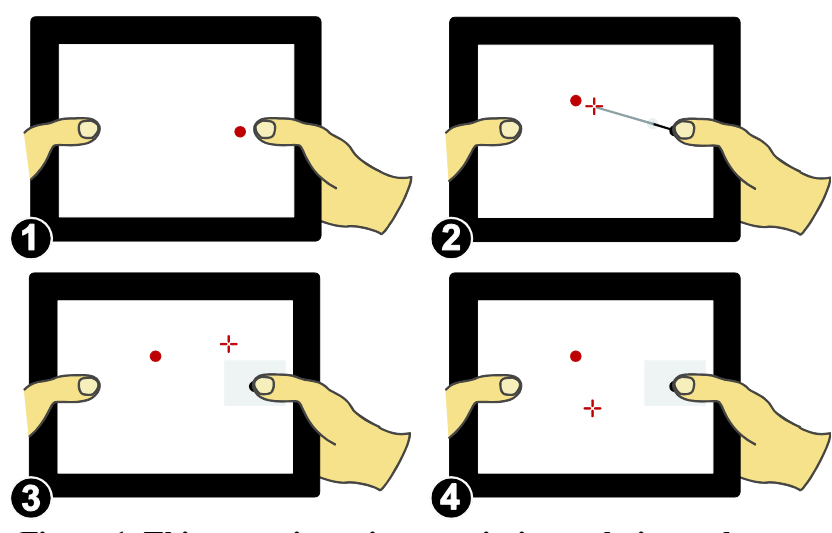

Figure 1. This paper investigates pointing techniques that are performed without losing the tablet grip: (1) direct touch (2) inverse cursor (3) touchPad (4) miniature area.

location where a target is displayed. If the direct touch is performed with the hands that hold a tablet in a symmetric bimanual grip, the thumb can execute direct touch on the front of the device and a finger does it on the back side. Direct touch is known to cause occlusion problems with touchscreens. This problem is addressed through back-ofdevice pointing proposed by Wigdor et al. [20]. Moreover, direct touch can make it difficult to access the center of tablets that are held with both hands $[12,21]$.

\section{(2) Inverse cursor}

The inverse cursor technique refers to MagStick [14] and Large Touch [10], which were developed for one-handed phone interactions. Similar to both approaches, the inverse cursor is controlled by setting an initial anchor point through a touch; and a drag gesture from that initial touch point pulls a cursor in the opposite direction. While the distance the cursor is moved using MagStick is similar to the length of the drag gesture; Large Touch translates the drag gesture to a larger cursor movement to overcome accessibility problems in one-handed phone interaction. The amount of movement of the inverse cursor is adaptive to allow for the entire interaction area to be always accessible when interacting with the hand that grasps the device. The cursor motion depends on the position of the start of the drag gesture. As the cursor movement is defined by an inverse digit movement, dragging the digit to the very outer touchscreen boarder will move the cursor to the touchscreen's very outer opposite edge. This ensures that every screen position is accessible.

\section{(3) TouchPad}

The touchPad technique is a virtual reference to physical touchpads that are commonly built in laptop computers. Researchers found that there are many ways to hold a tablet device [13, 19]; and Cheng et al. [3] proposed placing GUI components (such as virtual keyboards) where people grasp a tablet. Accordingly, our touchPad appears after an initial touch. A drag gesture on the touchPad moves a cursor relatively. For target acquisition using the touchPad, several drag gestures may be required to point on a target. Thus, a release cannot be used to confirm a target acquisition to 
finish the selection. We have chosen a tap gesture as confirmation for target selection. TouchPad is the only relative pointing technique in our experiment. Relative pointing is known to be slower but more precise than absolute pointing techniques, such as direct touch $[4,7]$.

\section{(4) Miniature area}

The principle of our miniature area is to provide a miniature representation of the entire screen that is accessible using the grasping hand. The approach is inspired by ThumbSpace [8], the Sliding-screen [10], and the ARC-Pad [11]. While the $A R C$-Pad uses a mobile phone as a physical one-to-one representation of a large display; our miniature area is a virtual miniature one-to-one representation of the tablet's interaction area. Unlike ThumbSpace and Sliding-screen, which require defining the interaction area size with a drag gesture; miniature area does not require an extra interaction step to size the interaction area. The miniature display representation is pre-defined in size and is dynamically placed through a touch gesture. That is similar to the adaptive placement of the touchPad and ensures that the miniature area is always well accessible aiming for faster interaction. Unlike the touchPad that requires a tap gesture to confirm a target selection process, the miniature area in more immediate. Target acquisition is realized through touching the miniature area at the position that is mapped to the corresponding target position on the tablet's display. The position on the large screen that a touch on the miniature area is referring to is visualized through a cursor. Drag gestures allow for moving a cursor. A touch release confirms the target acquisition and ends the selection process.

\section{EXPERIMENT}

To provide foundational knowledge about grasp-based pointing on tablets, and to help designers to understand the human factors, we compared four pointing techniques in a controlled experiment. We evaluate the pointing techniques to determine their performance as well as perceived effort and usability. Performance provides insights in target accessibility and selection time. Effort and usability help understanding which pointing technique is preferred for what reason.

\section{Design}

Our study had a $4 \times 2 \times 2 \times 3$ within subject design with the independent variables pointing technique (direct touch, inverse cursor, touchPad, miniature area), hand (right, left), device side (front-, back-of-device), and target size (5, 7, $10 \mathrm{~mm})$. The targets were arranged in a 10x7 grid that was equally distributed over the touchscreen, excluding 2 positions of "start" buttons at the vertical center of both most left and right outer $\mathrm{x}$-positions. One target per size appeared per target position in each condition. Thus, 204 targets had to be selected (or rejected if not accessible) per condition, resulting in 3264 targets per participant. The dependent variables were target selection time, selection effectiveness, perceived effort, and usability. Touch events (for each pointing technique, hand, device side, target size, and target position) were recorded in logfiles. Perceived effort (for each pointing technique and each device side) was measured using the SMEQ scale, because it is known to be very sensitive with small sample sizes [15]. The usability of each pointing technique was recorded using the AttrakDiff questionnaire.

\section{Participants}

We recruited 14 right-handed participants (6 females) with different academic backgrounds, such as computer science, history of art, and media design. The participants had an average age of 29.6 years $(\mathrm{SD}=4.3)$.

\section{Apparatus}

A tablet sandwich was used to present the experimental task and to record logfiles. It consisted of two tablet devices glued with their rear sides together and connected via Bluetooth. This allowed for sensing touch events on the back of the apparatus and to update the GUI of the device at the front accordingly. The resolution of the screen was $1280 \times 742$ pixels (without bottom menu bar) with a size of $21.7 \mathrm{~cm} \times 13.6 \mathrm{~cm}$

\section{Procedure}

For each pointing technique, we asked the participants to select targets that appeared after pressing a "start" button as fast and precise as possible. During the tasks, the participants were holding the apparatus in landscape format with two hands in a symmetric grip. Each pointing technique was used on both device sides using one hand after the other. If a target by any reason was perceived to be not easily selectable, the participants were asked to press a "cancel" button. We randomized the order of the 16 conditions. Each participant was asked to perform the tasks using each condition after a short training phase. After completing a condition, the participants filled the SMEQ and the AttrakDiff questionnaires. The experiment was split into two sessions. Each session lasted between 1.5 and 2 hours. When all conditions were completed, a demographic questionnaire was filled.

\section{RESULTS}

Our results contain effectiveness (measured as percentage of targets that could successfully be selected), efficiency (measured as task completion time), selection time per target position, number of attempts to select a target, perceived effort, and usability.

\section{Effectiveness}

Effectiveness represents the target accessibility for the different pointing techniques. If the participants were not able to select a target, for instance because they could not reach it, they could skip that task with a "cancel" button. The cancelled selection tasks per pointing technique are presented in Table 1. 


\begin{tabular}{|l|c|c|c|c|}
\hline $\begin{array}{l}\text { pointing } \\
\text { technique }\end{array}$ & $\begin{array}{c}\text { direct } \\
\text { touch }\end{array}$ & $\begin{array}{c}\text { inverse } \\
\text { cursor }\end{array}$ & touchPad & $\begin{array}{c}\text { mini. } \\
\text { area }\end{array}$ \\
\hline & \multicolumn{4}{|c|}{ Front of the device } \\
\hline right hand & $50.95 \%$ & $0.00 \%$ & $0.00 \%$ & $0.00 \%$ \\
\hline left hand & $51.65 \%$ & $0.00 \%$ & $0.00 \%$ & $0.00 \%$ \\
\hline \multicolumn{5}{|c|}{ Back of the device } \\
\hline right hand & $52.71 \%$ & $0.00 \%$ & $0.00 \%$ & $0.09 \%$ \\
\hline left hand & $54.34 \%$ & $0.17 \%$ & $0.04 \%$ & $1.57 \%$ \\
\hline
\end{tabular}

Table 1. Cancelled selections tasks per pointing technique.

While the target accessibility for inverse cursor, touchPad, and miniature area was $100 \%$ on the front side and above $98 \%$ on the back of the device. Pointing with direct touch was cancelled in more than half of the cases. As it is expected, targets that are further away from the grasping hand are not reachable via direct touch. The target positions that were accessible in at least $90 \%$ of the attempts cover $37 \%$ of the front sided interaction area for each hand as well as $34 \%$ of the back of the tablet using the left hand and $30 \%$ using the right hand, as shown in Figure 2.

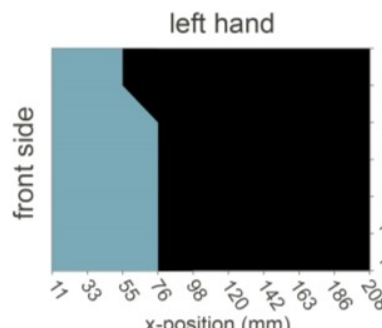
x-position ( $\mathrm{mm}$ )

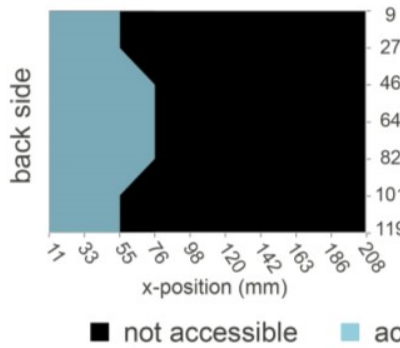

for direct touch
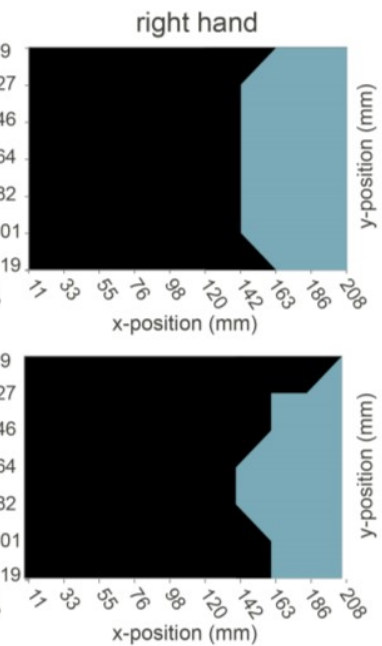

accessible

(in at least $90 \%$ of the tries)
Figure 2. Locations of the targets that are accessible using direct touch.

\section{Efficiency}

Efficiency is measured as target selection time for the targets that were successfully selected. The selection tasks that were cancelled are not considered in this analysis. We removed 528 of the 45.696 tasks that were more than three standard deviations from the mean and took longer than 8.644s [18]. The average selection times (Mean) and the standard deviations (SD) are presented in Table 2.

A Kolmogorov-Smirnov-Test shows that our data is normally distributed. Mauchly's test indicated that the assumption of sphericity has been violated for the interaction effects technique * hand $(\mathrm{p}<.001)$.

\begin{tabular}{|c|c|c|c|c|}
\hline & \multicolumn{4}{|c|}{ pointing technique } \\
\hline & $\begin{array}{l}\text { direct } \\
\text { touch }\end{array}$ & $\begin{array}{l}\text { inverse } \\
\text { cursor }\end{array}$ & touchPad & $\begin{array}{c}\text { mini. } \\
\text { area }\end{array}$ \\
\hline Mean & $1.28 \mathrm{~s}$ & $2.93 \mathrm{~s}$ & $2.53 \mathrm{~s}$ & $1.97 \mathrm{~s}$ \\
\hline \multirow[t]{3}{*}{ SD } & $0.74 \mathrm{~s}$ & $1.29 \mathrm{~s}$ & $0.92 \mathrm{~s}$ & $0.87 \mathrm{~s}$ \\
\hline & \multicolumn{2}{|c|}{ device side } & \multicolumn{2}{|c|}{ hand } \\
\hline & front & back & left & right \\
\hline Mean & $2.06 \mathrm{~s}$ & $2.57 \mathrm{~s}$ & $2.40 \mathrm{~s}$ & $2.22 \mathrm{~s}$ \\
\hline \multirow[t]{3}{*}{ SD } & $1.06 \mathrm{~s}$ & $1.17 \mathrm{~s}$ & $1.15 \mathrm{~s}$ & $1.13 \mathrm{~s}$ \\
\hline & \multicolumn{4}{|c|}{ target size } \\
\hline & $5 \mathrm{~mm}$ & & & $10 \mathrm{~mm}$ \\
\hline Mean & $2.51 \mathrm{~s}$ & & & $2.13 \mathrm{~s}$ \\
\hline SD & $1.24 \mathrm{~s}$ & & & $1.03 \mathrm{~s}$ \\
\hline
\end{tabular}

Table 2. Mean and SD for target selection time in seconds.

Therefore degrees of freedom were corrected using Greenhouse-Geisser estimates of sphericity for the respective tests. There was a significant main effect of pointing technique on the task completion time $\left(F_{3,36}=124.24, p<.001\right)$. Bonferroni corrected pair-wise ttests revealed significant differences between the four pointing techniques ( $p=.007$ for inverse cursor vs. touchPad and $p<.001$ for all other comparisons, see Figure $3)$. Similarly, hand $\left(F_{1,12}=20.77, p=.001\right)$, device side $\left(F_{1,12}=60.64, \quad p<.001\right.$, Figure 4$)$, and target size $\left(F_{2,24}=167.80, p<.001\right)$ had a significant effect on the task completion time. Bonferroni corrected pair-wise t-tests revealed significant differences between the three target sizes $(p<.001$, see Figure 5$)$. We found interaction effects for technique * device side $\left(F_{3,36}=10.33, p<.001\right)$, hand * device side $\left(F_{1,12}=6.88, p=.022\right.$, Figure 6$)$, and technique $*$ target size $\left(F_{6,72}=21.22, p<.001\right)$. In contrast, there were neither significant interaction effects for technique * hand $\left(F_{1.27,15.19}=0.76, p=.43\right)$, hand $*$ target size $\left(F_{2,24}=0.15\right.$, $\mathrm{I}=.87)$, nor for device side * target size $\left(\mathrm{F}_{2,24}=2.98, p=.07\right)$.

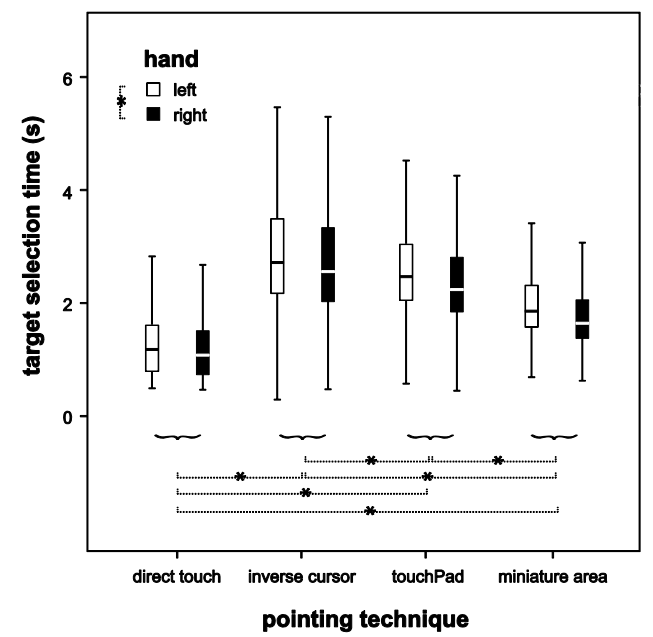

Figure 3. Selection times over pointing technique per hand and sign. differences (*). 


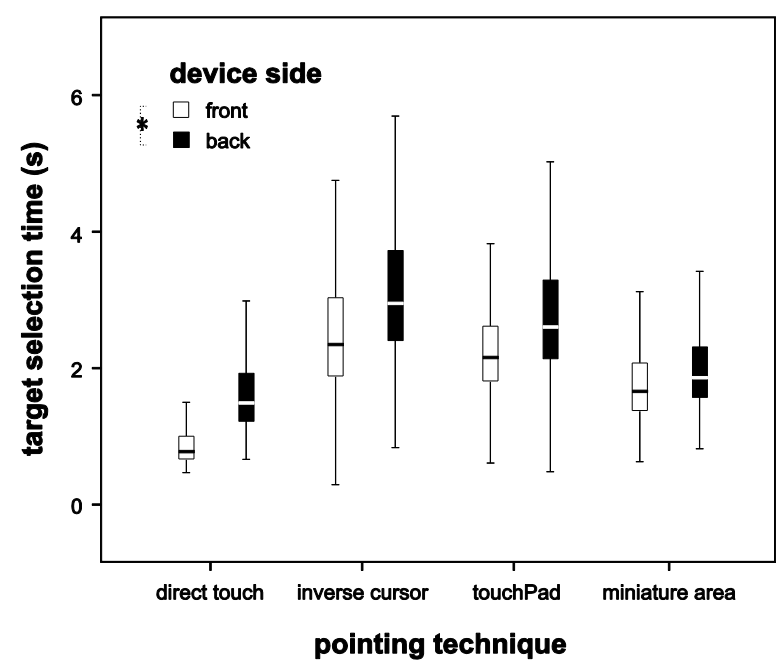

Figure 4. Selection times over pointing techniques per device side.

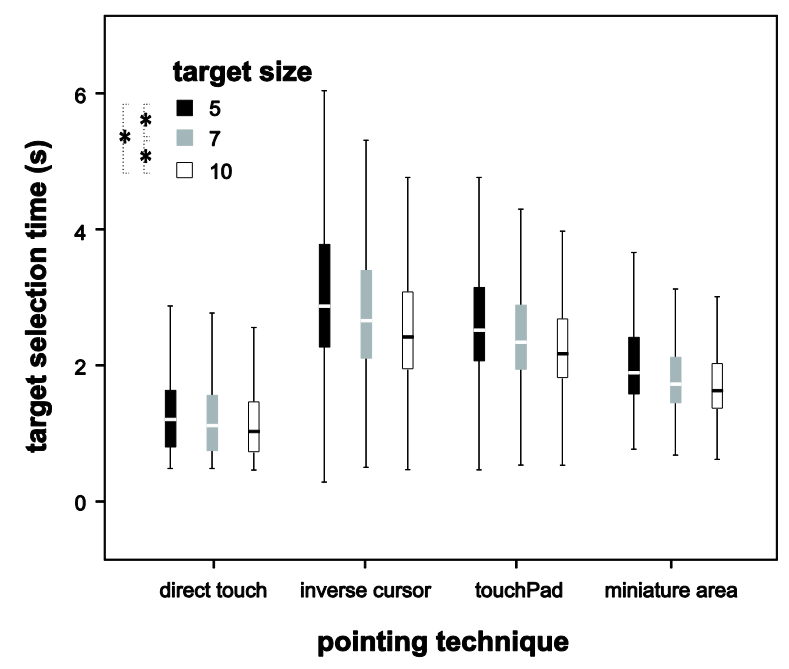

Figure 5. Selection times over pointing techniques per target size.

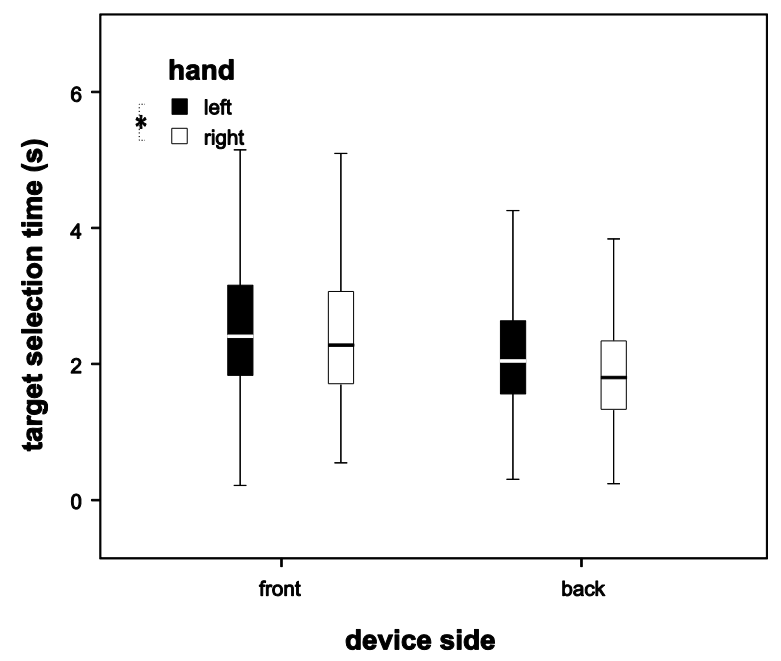

Figure 6. Selection times over device side per hand.

\section{Selection time per target position}

Selection time for each target position was calculated if the target was at least successfully selected in at least $90 \%$ of the attempts. Otherwise the target position was identified to be not accessible. The median time needed to select a target at a certain position is presented in Figure 7. Direct touch just allows for accessing targets that are located close to the hand that is selecting it. The other three techniques allow accessing the entire interaction area on both, the front and the back of the device. As shown in Figure 3; the inverse cursor has the lowest performance. Selection time increases the further the target is from the selecting hand for the inverse cursor and the touchPad technique. The miniature area results in an almost constant time across the screen and also in the highest selection performance over the entire interaction area on both device sides.

\section{Number of attempts per target selection}

We counted how many attempts participants needed to successfully select a target. The numbers of attempts are given by the number of touch downs/releases. Cancelled attempts were ignored for this calculation. We removed all tasks that were more than three standard deviations from the mean and took more than 10.4 attempts [18]. The average number of attempts (Mean) and the standard deviations (SD) for each pointing technique, device side, hand, and target size are presented in Table 3.

Again, a Kolmogorov-Smirnov-Test shows that our data is normally distributed, and Mauchly's test indicated that the assumption of sphericity has been violated for the main effect technique $(p<.001)$ as well as for the interaction effects technique * hand $(p<.001)$, and technique * device side $(p=.001)$. Therefore degrees of freedom were corrected using Greenhouse-Geisser estimates of sphericity for the respective tests.

\begin{tabular}{|c|c|c|c|c|}
\hline & \multicolumn{4}{|c|}{ Pointing technique } \\
\hline & $\begin{array}{l}\text { direct } \\
\text { touch }\end{array}$ & $\begin{array}{l}\text { inverse } \\
\text { cursor }\end{array}$ & touchPad & $\begin{array}{c}\text { mini. } \\
\text { area }\end{array}$ \\
\hline Mean & 1.2 & 1.2 & 3.1 & 1.1 \\
\hline \multirow[t]{3}{*}{ SD } & 0.6 & 0.6 & 1.1 & 0.4 \\
\hline & \multicolumn{2}{|c|}{ device side } & \multicolumn{2}{|c|}{ hand } \\
\hline & front & back & left & right \\
\hline Mean & 1.7 & 1.7 & 1.7 & 1.7 \\
\hline \multirow[t]{3}{*}{ SD } & 1.2 & 1.2 & 1.2 & 1.2 \\
\hline & \multicolumn{4}{|c|}{ target size } \\
\hline & $5 \mathrm{~mm}$ & & & $10 \mathrm{~mm}$ \\
\hline Mean & 1.8 & & & 1.7 \\
\hline SD & 1.2 & & & 1.1 \\
\hline
\end{tabular}

Table 3. Mean and SD for number of attempts to select a target. 


\section{front-of-device pointing}
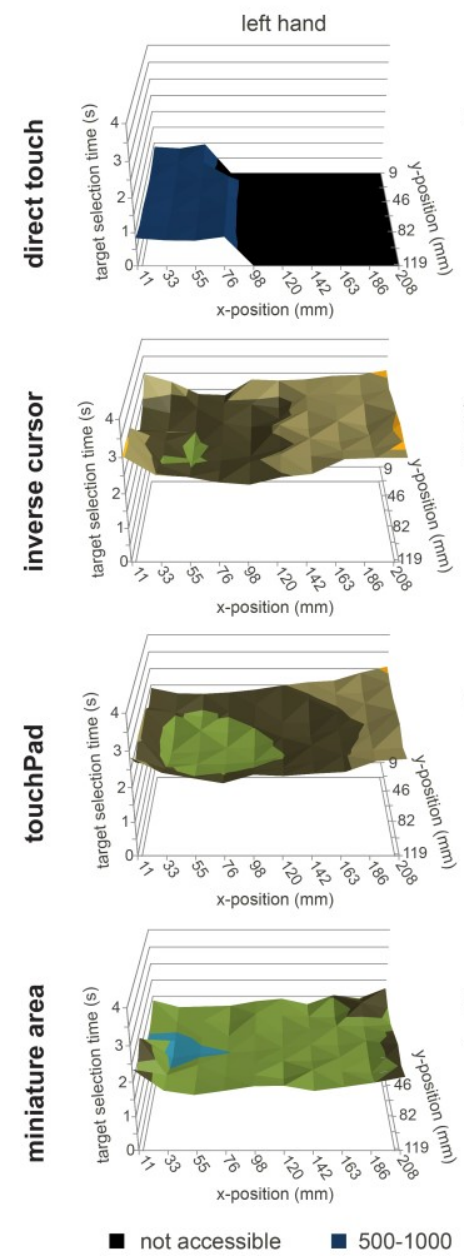

right hand
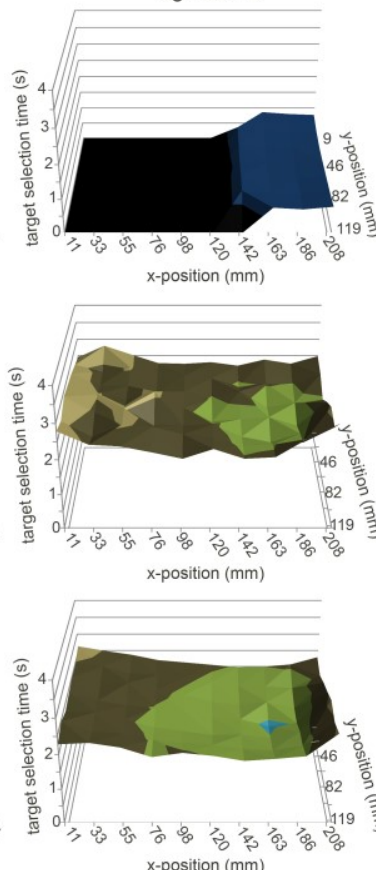

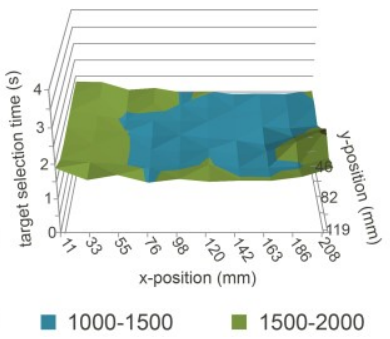

back-of-device pointing
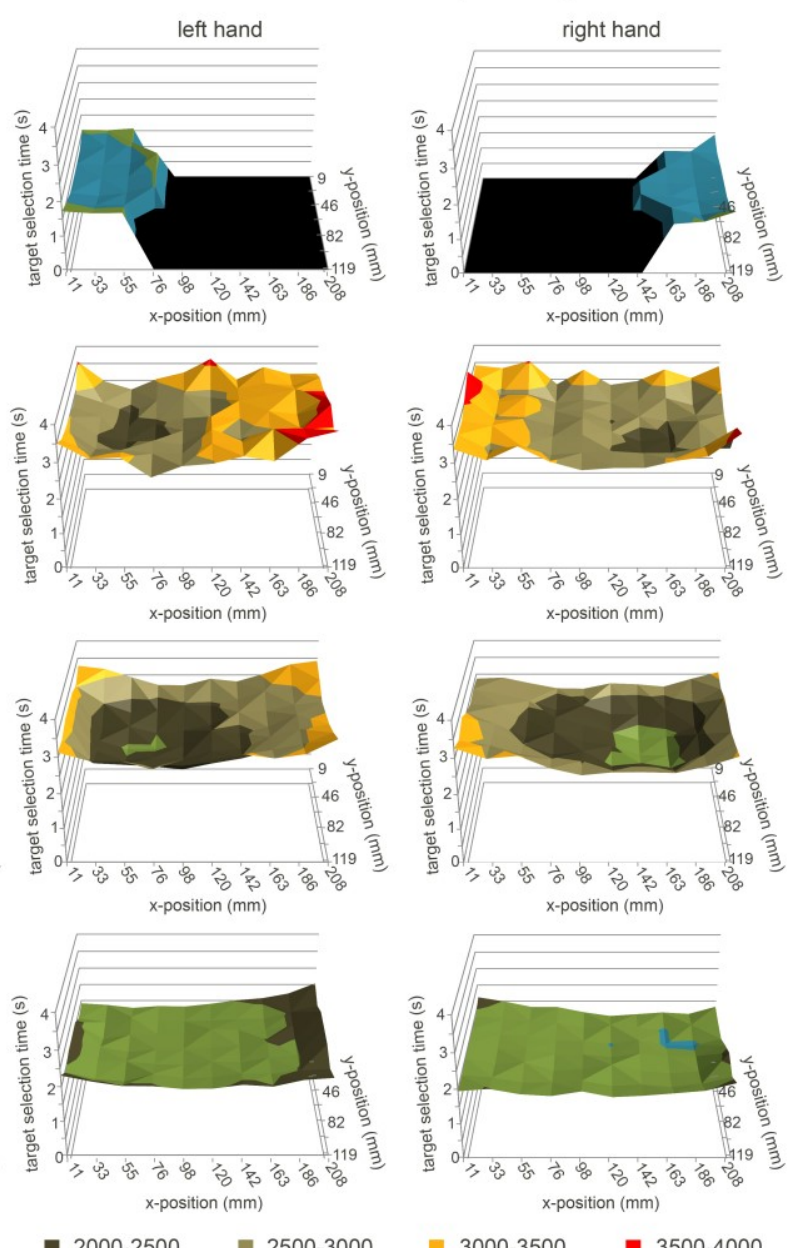

- 2000-2500 2500-3000

- 3000-3500

- $3500-4000$

Figure 7. Target selection time (median) for each target position at the front and the back of a tablet (counted in mm from the top left). If the target could not be selected in $\mathbf{9 0 \%}$ of the attempts in a certain position, this position is labelled as not accessible.

There was a significant main effect of the pointing technique on the number of attempts participants needed to select a target $\left(F_{1.07,12.87}=175.89, \quad p<.001\right.$. Bonferroni corrected pair-wise t-tests revealed significant differences between inverse cursor and touchPad $(p<.001)$, direct touch and touchPad $(p<.001)$, direct touch and miniature area $(p=.002)$, as well as touchPad and miniature area $(p<.001)$, see Figure 8. There were no significant main effects for hand $\left(F_{1,12}=0.001, p=.97\right)$ or device side $\left(F_{1,12}=0.03, p=.87\right)$. Target size $\left(F_{2,24}=33.17, p<.001\right)$ had a significant effect on the number of attempts. Bonferroni corrected pair-wise t-tests revealed significant differences between all target sizes ( $p=.047$ for $5 \mathrm{~mm}$ vs. $7 \mathrm{~mm}, p<.001$ for $5 \mathrm{~mm}$ vs. $10 \mathrm{~mm}$, and $p=.001$ for $7 \mathrm{~mm}$ vs. $10 \mathrm{~mm}$ ). We found significant interaction effects for technique * target size $\left(F_{6,72}=11.14, p<.001\right)$ and device side * target size $\left(F_{2,24}=8.93, \quad p<.001\right)$. There were neither significant interaction effects for technique $*$ hand $\left(F_{1.53,18.35}=0.52\right.$, $p=.07)$, technique $*$ device side $\left(F_{1.91,22.89}=2.73, p=.09\right.$, Figure 9$)$, hand $*$ device side $\left(F_{1,12}=0.13, p=0.91\right)$, nor for hand $*$ target size $\left(F_{2,24}=0.26, p=.97\right)$.

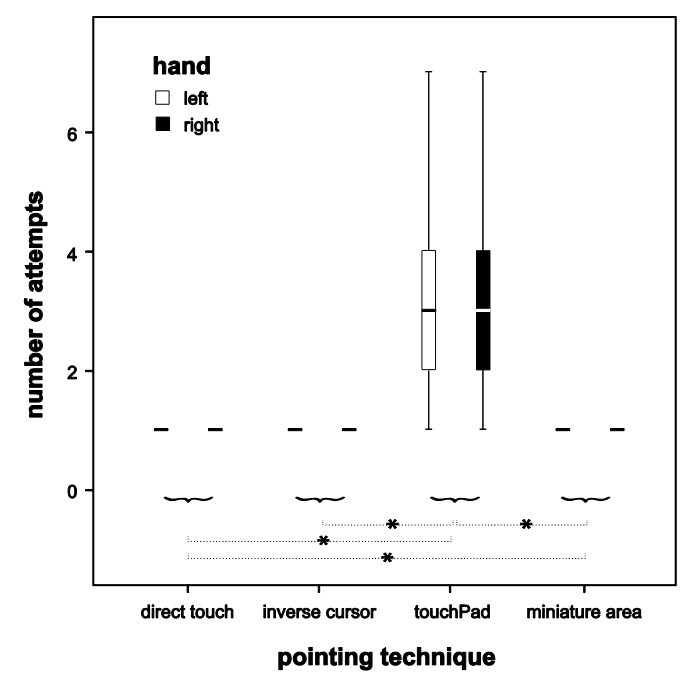

Figure 8. Number of attempts over pointing technique per hand and sign. differences $(*)$. 


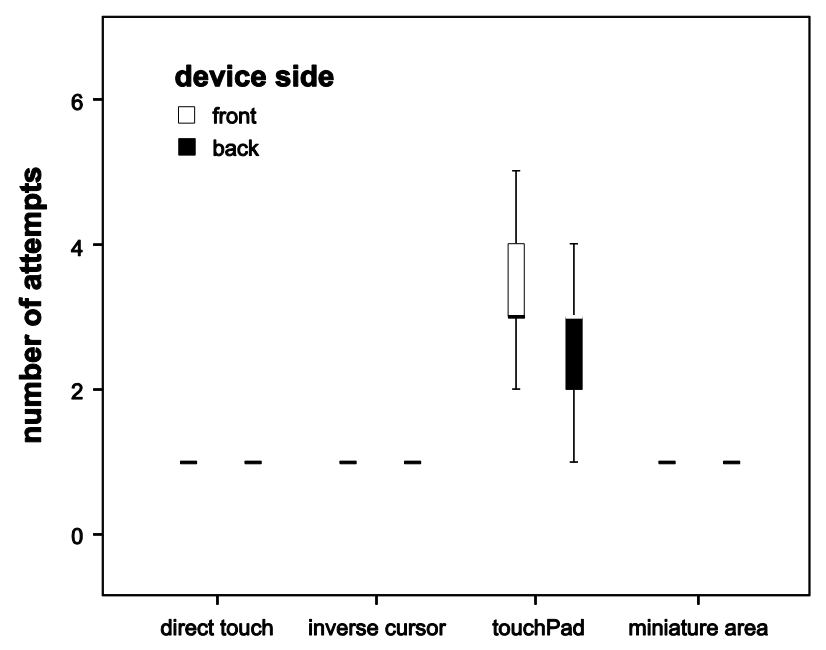

pointing technique

Figure 9. Number of attempts over pointing techniques per device side.

\section{Perceived effort}

The perceived effort was measured using the SMEG on a scale from 0 (no effort) to 220 (high effort). The average perceived effort (Mean) and the standard deviations (SD) as well as the according effort description are presented in Table 4.

\begin{tabular}{|l|c|c|c|c|}
\hline $\begin{array}{l}\text { Pointing } \\
\text { techniques }\end{array}$ & $\begin{array}{c}\text { direct } \\
\text { touch }\end{array}$ & $\begin{array}{c}\text { inverse } \\
\text { cursor }\end{array}$ & touchPad & $\begin{array}{c}\text { miniature } \\
\text { area }\end{array}$ \\
\hline Mean & 81.3 & 104.8 & 54.9 & 49.2 \\
\hline SD & 14.0 & 14.0 & 14.0 & 14.0 \\
\hline Description & fairly hard & pretty hard & a bit hard & a bit hard \\
\hline Device side & \multicolumn{2}{|c|}{ front } & \multicolumn{2}{c|}{ back } \\
\hline Mean & \multicolumn{2}{|c|}{65.3} & \multicolumn{2}{c|}{79.8} \\
\hline SD & \multicolumn{2}{|c|}{10.8} & \multicolumn{2}{c|}{10.8} \\
\hline Description & \multicolumn{2}{|c|}{ fairly hard } & \multicolumn{2}{c|}{ fairly hard } \\
\hline
\end{tabular}

Table 4. Perceived effort with Mean, SD, and description.

A repeated measure ANOVA yielded a significant different perceived effort for pointing technique $\left(F_{3,80}=7.2, p<.001\right)$. For device side it was shown that interacting on the front was perceived significantly easier $\left(F_{1,92}=7.9, p=.006\right)$. Moreover, we found an interaction effect for pointing technique $*$ device side $\left(F_{3,81}=3.1, p=.030\right)$.

While Bonferroni-corrected pairwise comparisons (see Figure 10) showed that the inverse cursor technique was perceived to be significantly harder than the touchPad $(p=.021)$ as well as than the miniature area $(p<.001)$, no significant difference was found between direct touch and any other technique (direct touch vs. inverse cursor: $p=1.000$, direct touch vs. touchPad: $p=.221$, and direct touch vs. miniature area: $p=.336$ ). Furthermore, we found no significant difference between the touchPad and the miniature area $(p=1.000)$.

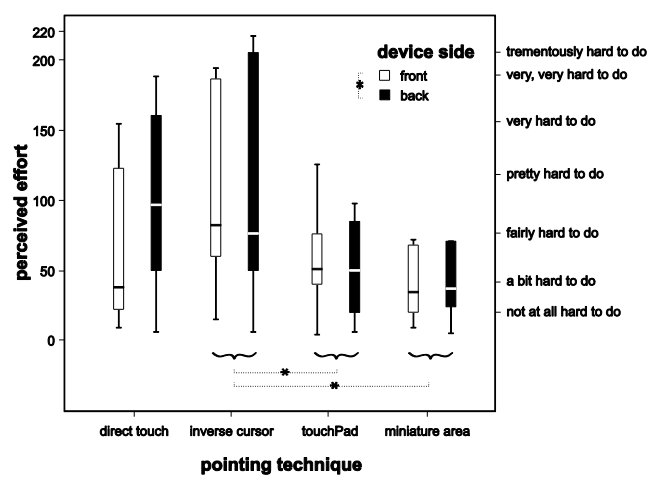

Figure 10. Perceived effort over pointing techn. per device side.

\section{Usability}

The four sub-scales of the AttrakDiff questionnaire, which describe usability, are pragmatic qualities, the hedonic quality of identification, the hedonic quality of stimulation, and global attractiveness. All four scales have as minimum 1 and as maximum 7, while high values refer to high qualities. The average usability qualities (Mean) and the standard deviations (SD) are presented in Table 5.

\begin{tabular}{|c|c|c|c|c|}
\hline $\begin{array}{l}\text { Pointing } \\
\text { techniques }\end{array}$ & $\begin{array}{l}\text { direct } \\
\text { touch }\end{array}$ & $\begin{array}{l}\text { inverse } \\
\text { cursor }\end{array}$ & touchPad & $\begin{array}{c}\text { miniature } \\
\text { area }\end{array}$ \\
\hline & \multicolumn{4}{|c|}{ Pragmatic quality } \\
\hline Mean & 4.8 & 3.9 & 5.2 & 5.2 \\
\hline \multirow[t]{2}{*}{ SD } & 0.2 & 0.4 & 0.2 & 0.3 \\
\hline & \multicolumn{4}{|c|}{ Hedonic quality: Identification } \\
\hline Mean & 4.1 & 4.2 & 4.4 & 4.7 \\
\hline \multirow[t]{2}{*}{ SD } & 0.2 & 0.2 & 0.2 & 0.2 \\
\hline & \multicolumn{4}{|c|}{ Hedonic quality: Stimulation } \\
\hline Mean & 4.4 & 4.2 & 4.4 & 4.4 \\
\hline \multirow[t]{2}{*}{ SD } & 0.3 & 0.3 & 0.3 & 0.3 \\
\hline & \multicolumn{4}{|c|}{ Attractiveness } \\
\hline Mean & 4.6 & 3.8 & 4.9 & 5.2 \\
\hline SD & 0.3 & 0.3 & 0.2 & 0.3 \\
\hline \multirow[t]{2}{*}{ Device side } & \multicolumn{2}{|c|}{ front } & \multicolumn{2}{|c|}{ back } \\
\hline & \multicolumn{4}{|c|}{ Pragmatic quality } \\
\hline Mean & \multicolumn{2}{|c|}{5.0} & \multicolumn{2}{|c|}{4.6} \\
\hline \multirow[t]{2}{*}{ SD } & & & & \\
\hline & \multicolumn{4}{|c|}{ Hedonic quality: Identification } \\
\hline Mean & \multicolumn{2}{|c|}{4.3} & \multicolumn{2}{|c|}{4.4} \\
\hline \multirow[t]{2}{*}{ SD } & & & & \\
\hline & \multicolumn{4}{|c|}{ Hedonic quality: Stimulation } \\
\hline Mean & & & & \\
\hline \multirow[t]{2}{*}{ SD } & & & & \\
\hline & \multicolumn{4}{|c|}{ Attractiveness } \\
\hline Mean & \multicolumn{2}{|c|}{4.9} & \multicolumn{2}{|c|}{4.4} \\
\hline SD & \multicolumn{2}{|c|}{0.2} & \multicolumn{2}{|c|}{0.3} \\
\hline
\end{tabular}

Table 5. Mean values and SD for the AttrakDiff sub-scales. 


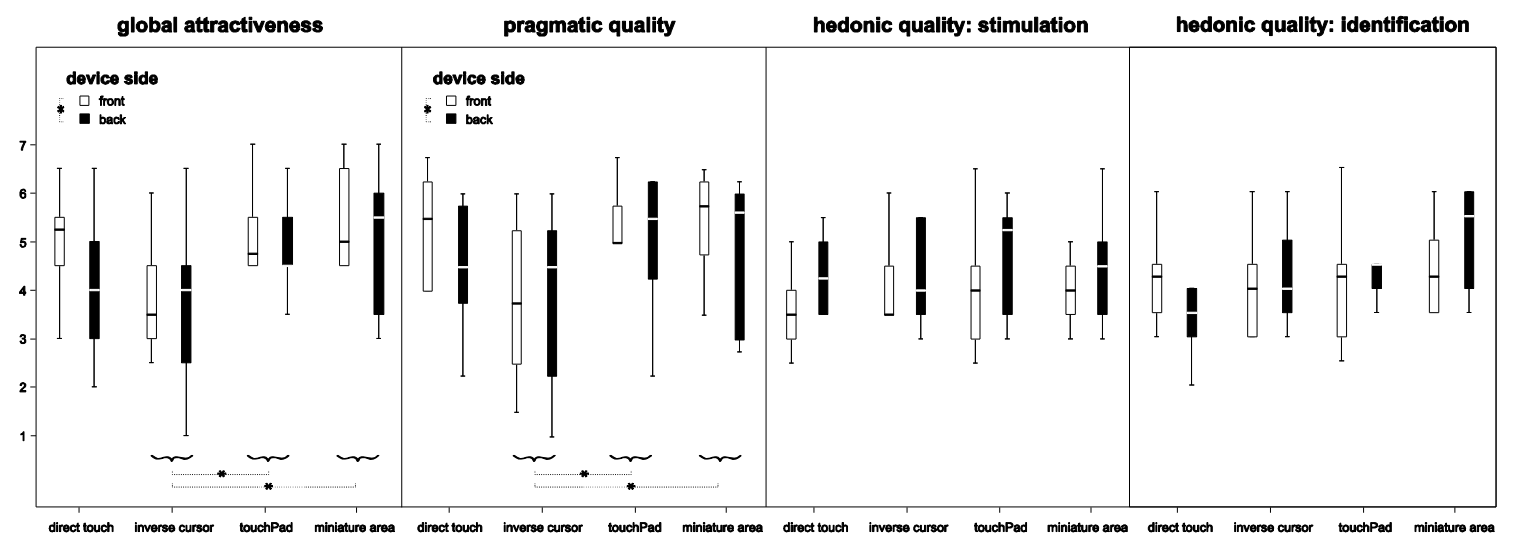

pointing technique

Figure 11: Usability scales: global attractiveness, pragmatic quality, hedonic quality stimulation, hedonic quality identification to rate the four pointing techniques from $1=$ low rating to $7=$ high rating.

A MANOVA yielded significantly different ratings per pointing technique for the global attractiveness and the pragmatic qualities (global attractiveness: $F_{3,114}=9.2$, $p<.001$, pragmatic quality: $\left.F_{3,114}=10.2, \quad p<.001\right)$. Both hedonic qualities, identification and stimulation were not rated differently (identification: $F_{3,114}=2.5, \quad p=.071$, stimulation: $\left.F_{3,144}=1.8, \quad p=.155\right)$. Moreover, the attractiveness and the pragmatic qualities were rated significantly different for both, pointing on the front and the back of the device (global attractiveness: $F_{1,10}=6.7, p=.023$, pragmatic quality: $F_{1,10}=8.9, p=.011$ ). Again, the hedonic qualities were not rated differently for both device sides (identification: $F_{3,114}=1.4, p=.517$, stimulation: $F_{3,144}=4.5$, $p=.054)$. No interaction effect was found between the pointing techniques and device side (global attractiveness: $F_{3,114}=1.3, p=.291$, pragmatic quality: $F_{3,114}=1.4, p=.268$, stimulation: $F_{3,144}=2.2, p=.103$, identification: $F_{3,114}=2.0$, $p=.128$ ).

For attractiveness, Bonferroni-corrected pairwise comparisons (see Figure 11) showed that the inverse cursor technique received significantly lower ratings than the miniature area $(p=.003)$ and the touchPad $(p=.006)$. According pragmatic qualities, Bonferroni corrected posthoc tests showed again that the inverse cursor was rated worse than the miniature area $(p<.001)$ as well as than the touchPad $(p=.002)$.

\section{Comments}

During the experiment, participants gave additional comments about their experience with the different pointing techniques. Four participants appreciated that targets were not occluded when interacting on the back of the device. Targets are always occluded when pointing via direct touch on the front side but also through the other three techniques if the targets were located on bottom positions where the thumb is rested while holding the device. One participant mentioned that a tap was sometimes misinterpreted as a drag using the touchPad.

\section{DISCUSSION}

Analyzing the collected data, we found that only $37 \%$ of the front and $32 \%$ of the back of tablets is accessible using direct touch with one hand. Even with two hands, only $74 \%$ of the front and $64 \%$ of the back. While direct touch is the fastest pointing technique, we found that it is only usable for areas at the screen's border. Among the other pointing technique, the miniature area is $28.4 \%$ faster than the touchPad and $48.7 \%$ faster than the inverse cursor. In contrast to the other techniques, the target selection time varies little across the screen using the miniature area. Looking at the subjective measures of all four techniques, using the miniature area resulted in the lowest perceived effort and the highest score on all sub-scales of the AttrakDiff questionnaire even in comparison with direct touch.

Our results are in line with Odell et al. [12] as well as with Wolf et al. [21] who found that the center areas of tablet touchscreens and for back-of-device interaction are not accessible with direct touch. Moreover, we have shown that direct touch on the back of the device results in the same problem. While Kim et al. [10] found that their inverse cursor Large Touch is as fast as direct touch when pointing on mobile phones, our results are contradictory to these but in line with those of Roudaut et al. [14] who found that direct touch is faster than the inverse cursor technique using mobile phones. The miniature areas ThumbSpace [8] and Sliding-screen [10] implemented for mobile phones were slower than direct touch, which is in line with our findings using the miniature area on the front as well as on the back of tablet devices. Moreover, our miniature area is (similar to the ARC-Pad) faster than relative pointing, which is represented through the touchPad technique in our study. While Hasan et al. [7] found that relative pointing performs better than direct touch for one-handed back-ofphone interaction in terms of target selection time as well as accuracy, we found like Cockburn et al. [4] that direct touch is faster than relative pointing, using our touchPad on 
both the front as well as the back of a tablet. In contrast to the findings of Cockburn et al. [4] our findings do not show an increase in the number of attempts for small targets.

In light of our results, designers of applications for tablet devices currently have two options when arranging interactive controls in a landscape UI. They can either use the whole screen while forcing the user to choose another grip or they can arrange all interactive controls on the $37 \%$ on the front and $32 \%$ on the back of tablets near each vertical screen's border. An indirect pointing technique such as the miniature area could offer a third option and make the whole screen easily accessible. As all indirect techniques we considered are slower than direct touch, we propose a combination of a miniature area with direct touch. As the miniature area performs equally well on the front as on the back a viable option is to combine direct touch on the front with the miniature area on the back. This approach has the advantage that a dedicated pointing technique is used on each device side which helps to avoid confusion of the user. Furthermore, the whole screen becomes easily accessible and thus usable for designers.

\section{CONCLUSION AND FUTURE WORK}

In this paper, we investigated pointing on tablet devices. We compared four pointing techniques while participants held the device with both hands in landscape format. For this setup, we showed that only $74 \%$ on the front and $64 \%$ on the back of tablets can be easily reached using direct touch. Among the three alternatives, the miniature area, a virtual miniature one-to-one representation of the tablet's interaction area, is the fastest option. As this miniature area received the best subjective ratings, even compared to direct touch, we propose to combine direct touch on the front of tablet devices with the miniature area on the back. This combination would make the whole screen accessible and avoid mixing interaction techniques.

The conducted study focuses on one of the most common ways to hold tablet devices and as participants were comfortably seated the setup mimics the typical usage context. Future work should nonetheless investigate if the results hold true for other postures and situations. Furthermore, the screen size of the used device was 10.1"; and future work should investigate additional screen sizes. The fraction of the screen that is easily accessible will become even smaller for larger screens. It would be interesting to investigate if our results can be transferred to much larger tabletops where interaction is not restricted by hand size but by arm length.

\section{REFERENCES}

1. Baudisch, P., Chu, G. Back-of-device interaction allows creating very small touch devices. Proc. CHI'09.

2. Buschek, D., Schoenleben, O., Oulasvirta, A. Improving accuracy in back-of-device multitouch typing: a clusteringbased approach to keyboard updating. Proc. IUI'14.
3. Cheng, L., Liang, H., Wu, C., Chen, M. iGrasp: grasp-based adaptive keyboard for mobile devices. Proc. CHI'13.

4. Cockburn, A., Ahlström, D., Gutwin, C. Understanding performance in touch selections: Tap, drag and radial pointing drag with finger, stylus and mouse. Int. J. Hum.-Comput. Stud. 70, 3 (March 2012).

5. De Luca, A., von Zezschwitz, E., Nguyen, N. D. H., Maurer, M.-E., Rubegni, E., Scipioni, M. P., and Langheinrich, M.. Back-of-device authentication on smartphones. Proc. CHI'13.

6. Forlines, C., Wigdor, D., Shen, C., Balakrishnan, R. Directtouch vs. mouse input for tabletop displays. Proc. CHI'07.

7. Hasan, K., Yang, X.-D., Liang, H.-N., Irani, P. How to position the cursor?: an exploration of absolute and relative cursor positioning for back-of-device input. Proc. MobileHCI'12.

8. Karlson, A.K., Bederson, B.B. ThumbSpace: generalized onehanded input for touchscreen-based mobile devices. In Proc. INTERACT'07.

9. Kerr, D. R., Hotelling, S., Huppi, B. Q. 2010. U.S. Patent No. 7,800,592. Washington, DC: U.S. Patent and Trademark Office.

10. Kim, S., Yu, J., and Lee, G. Interaction techniques for unreachable objects on the touchscreen. Proc. OzCHI'12.

11. McCallum, D. C. and Irani, P. ARC-Pad: absolute+relative cursor positioning for large displays with a mobile touchscreen. Proc. UIST'09.

12. Odell, D., Chandrasekaran, V. Enabling comfortable thumb interaction in tablet computers: a Windows 8 case study. In Proceedings of the Human Factors and Ergonomics Society Annual Meeting, 2012.

13. Oulasvirta, A., Reichel, A., Li, W., Zhang, Y., Bachynskyi, M., Vertanen, K., Kristensson, P. O. Improving two-thumb text entry on touchscreen devices. Proc. CHI'13.

14. Roudaut, A., Huot, S., Lecolinet, E. TapTap and MagStick: improving one-handed target acquisition on small touchscreens. Proc. AVI'08.

15. Sauro, J., Dumas, J. S. Comparison of three one-question, post-task usability questionnaires. Proc. CHI'09.

16. Siek, K.A., Rogers, Y., Connelly, K.H. Fat finger worries: how older and younger users physically interact with PDAs. Proc. INTERACT'05.

17. Trudeau, M. B., Young, J. G., Jindrich, D. L., Dennerlein, J. T. Thumb motor performance varies with thumb and wrist posture during single-handed mobile phone use. Journal of biomechanics (2012).

18. Tukey, J. W. Exploratory data analysis. Reading, Ma'77.

19. Wagner, J., Huot, S., Mackay, W.E. BiTouch and BiPad: designing bimanual interaction for hand-held tablets. Proc. CHI'12.

20. Wigdor, D., Forlines, C., Baudisch, P., Barnwell, J., Shen, C. Lucid touch: a see-through mobile device. Proc. UIST'07.

21. Wolf, K., Schleicher, R., Rohs, M. Touch Accessibility on the Front and the Back of held Tablet Devices. Proc. EuroHaptics'14. 